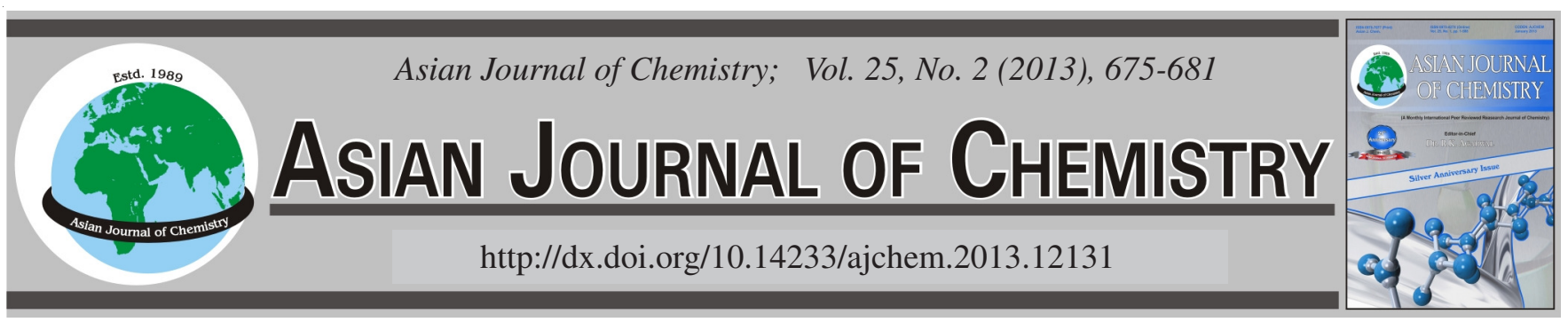

\title{
Study on the Fluorescence Characteristics of CdTe and Charged Coumarin-Contained Polymer Blending System
}

\author{
Xu Wang, Lichao Li, Deben Chen and Anyong Zhong*
}

College of Chemistry, Sichuan University, Chengdu 610064, P.R China

*Corresponding author: E-mail: zhongany@sina.com

\begin{abstract}
In this paper, a series of positively charged coumarin-contained polymer (C-CPA) were synthesized and a negatively charged CdTe quantum dots (CdTe QDs) protected by thioglycolic acid (TGA) was prepared. C-CPA and CdTe QDs were blended in mixed solution to build a blending system. The photoluminescent properties of both C-CPA and CdTe QDs were studied and the influence of the solvent on the size of CdTe QDs was studied as well. By controlling the proportions of the two mixtures, some regularly phenomena were found. As the proportion of CdTe QDs rising in the mixture, the fluorescence intensity of C-CPA declined; as the proportion of C-CPA rising, the fluorescence intensity of CdTe QDs rose significantly. These phenomena followed the fluorescence resonance energy transfer theory (FRET). The electrostatic force between the two mixtures caused the interaction between them. The study confirmed that an energy donor-acceptor pair was formed in this blending system.
\end{abstract}

Key Words: Blending, Fluorescence, Nanocomposites, Photophysics, Quantum dots.

\section{INTRODUCTION}

Semiconductor nanocrystals, also known as quantum dots (QDs), have attracted great fundamental and industrial interest in recent years ${ }^{1-3}$. Because of the quantum confinement effect ${ }^{4,5}$, the continuous energy band structure was changed into a discrete energy level structure with molecular characterization. The QDs, generally ranging from 1-10 nm in size, emits fluorescence when excited. Compared to organic dyes, semiconductor nanocrystals have showed some distinct characteristics, such as narrow and adjustable fluorescence emission peak, stable photochemical properties and high fluorescence efficiency and so on. Among them, the size-dependent photoluminescence (PL) is probably the most attractive characteristic.

For instance, CdSe or CdTe nanocrystals with photoluminescence varying from green to red can be obtained by a single synthetic route. These highly fluorescent nanocrystals have showed great applying potential in the field of thin film lightemitting devices ${ }^{6}$, nonlinear optical devices ${ }^{7}$, solar cells ${ }^{8,9}$, especially of biological labels ${ }^{10,11}$.

For the real applications of these highly fluorescent semiconductor nanocrystals, the inherently instable particles are generally stabilized using inert inorganic or organic materials as matrices. Although the researches of the functional materials based on semiconductor nanocrystals and polymer complex were often reported in recent years ${ }^{12-14}$, polymers were only taken as matrices to stabilize the semiconductor nanocrystals, the polymers themselves didn't have any contribution to the photoluminescence property in most of the researches. Pan et $a l .{ }^{15}$ indicated that the polymers were always used to make the semiconductor nanocrystals and polymer complex biocompatible and stable under various environments while the complex was used in the field of biological applications and they didn't improve the photoluminescence property of the complex. Cao et al..$^{14}$ believed that what the polymer contributed to the semi-conductor nanocrystals and polymer complex were its optical transparency, physical and chemical stability, tunable mechanical properties when the complex was used to fabricate light-emitting devices. Bertoni et al. ${ }^{16}$ referred that the photoluminescence property of the electroluminescent devices totally depended on the property of the semiconductor nanocrystals when the semiconductor nanocrystals and polymer complex was used to manufacture this kind of devices. These researches related to the usage of the polymer focused on its electroconductibility in their work. Even in the researches on the complex based on the semiconductor nanocrystals and luminescence polymers such as $\mathrm{PVK}^{17,18}$, the influences of the polymer to the photoluminescence property of the complex were not investigated in detail.

On the other hand, few researches on the influences and regularity of the interaction between the semiconductor nanocrystals and the polymer were reported. Sun et al. ${ }^{19}$ 
studied the variation of the photoluminescence property of the CdTe QDs capped with different materials before and after mixed with the polymer. But their work was tentative and lack systematicness. Wang et al. ${ }^{11}$ synthesized a kind of QD-labelled microsphere by self-assemble process and researched the variations of its photoluminescence property with addition of QD layers. But the work didn't mention the variation of fluorescence intensity before and after the addition of polymer and not yet explained whether the addition of polymer affects the photoluminescence property of quantum dots.

In order to study the interaction and the regularity between the semiconductor nanocrystals and the polymer, present experiment was designed to prepare a blending system by using a polymer containing fluorophore which was simply blended with a kind of semiconductor nanocrystal. The variations of the photoluminescence property of both the polymer and the semiconductor nanocrystal will be examined after blending and the influences caused by both sides of the mixture will be researched as well.

Though some researches have studied the interaction between the semiconductor nanocrystals and some kinds of conjugated polymers, the interaction between a polymer with side-chained fluorophore and the semiconductor nanocrystals has rarely been studied. Our experiment tried to build a strong connection between a polymer and quantum dots in the blending system with the electrostatic force. We wished to find some distinctive phenomena in the blending system.

A methyl methacrylate copolymer with side-chain containing coumarin was chosen as the polymer with fluorophore and CdTe QDs protected by TGA as the semiconductor nanocrystal. C-CPA contains a side-chain fluorophore which makes the polymer displaying certain photoluminescence property and the photoluminescence intensity related on the contents of 2-(dimethylamino) ethyl methacrylate (DM) in the main chain of C-CPA. TGA-capped CdTe QDs is a water-soluble semiconductor nanocrystal with good photoluminescence property and the particle size and florescence emission peak position of CdTe QDs can be adjusted by controlling the synthesis reaction time of the CdTe $\mathrm{QDs}^{20}$. On the other side, C-CPA carries positive charges when quaternized and TGA-capped
CdTe QDs carries negative charges on its surface. It is possible that both can form a set of energy donor-acceptor pair based on FRET if C-CPA and CdTe QDs can be coexisted in a certain system stably.

\section{EXPERIMENTAL}

Methyl methacrylate (MMA), 2-(dimethylamino)ethyl methacrylate (DM), 2-hydroxyethyl acrylate (HEA), $n$-butyl acrylate (BA), styrene ( $\mathrm{St}$ ), all of industrial grades were purchased from Beijing East Chemical Industry Factory, China and distilled before using. The initiator 2,2'-azo-bis-isobutyronitrile (AIBN, CR) was obtained from Shanghai No. 4 Reagent \& HV Chemical Co. Ltd. Tellurium powder (99.999 \%) was purchased from Shanghai Chemical Reagents Company. TGA (90\%), $\mathrm{CdCl}_{2} \cdot 2.5 \mathrm{H}_{2} \mathrm{O}$ (99\%), sodium borohydride (97\%) were obtained from Chengdu Kelong Chemical and Technology Reagents Ltd. Co., China.

Ultraviolet-visible (UV-VIS) adsorption spectra were measured by TU-1901 UV-VIS spectrophotometer. Fluorescence spectra were performed with Hitachi F-4500 spectrofluorimeter. Particle size distributions were measured by MAF5000 laser particle size analyzer.

Synthesis of positively charged C-CPA with different DM contents: By controlling the contents of DM in the methyl methacrylate copolymers (CPA), a series of CPA were synthesized by the reported method ${ }^{21}$. 3-(2-Benzothiazolyl)-6-chloromethyl-coumarin was synthesized with 2-hydroxybenzaldehyde, 2-aminothiophenol and malononitrile. The coumarin was blended with CPA under a condition of anhydrous and heated to synthesize a series of cationic copolymer. The basic structure of C-CPA was showed in Fig. 1-1. Based on the different contents of DM, this series of cationic copolymers were marked as $\mathrm{C}-\mathrm{CPA}_{1} \mathrm{C}-\mathrm{CPA}_{2}$ and $\mathrm{C}-\mathrm{CPA}_{3}$ just as shown in Table-1. The UV-VIS absorption peak was at $c a .365 \mathrm{~nm}$ and fluorescence emission peak was at about $458 \mathrm{~nm}$ (Fig. 1-2). The UV-VIS absorption peak of the products at $c a .365 \mathrm{~nm}$ proved that coumarin was grafted onto CPA successfully.

Synthesis of negatively charged TGA-capped CdTe QDs: Negatively charged TGA-capped CdTe QDs were synthesized in aqueous phase using the method reported by the

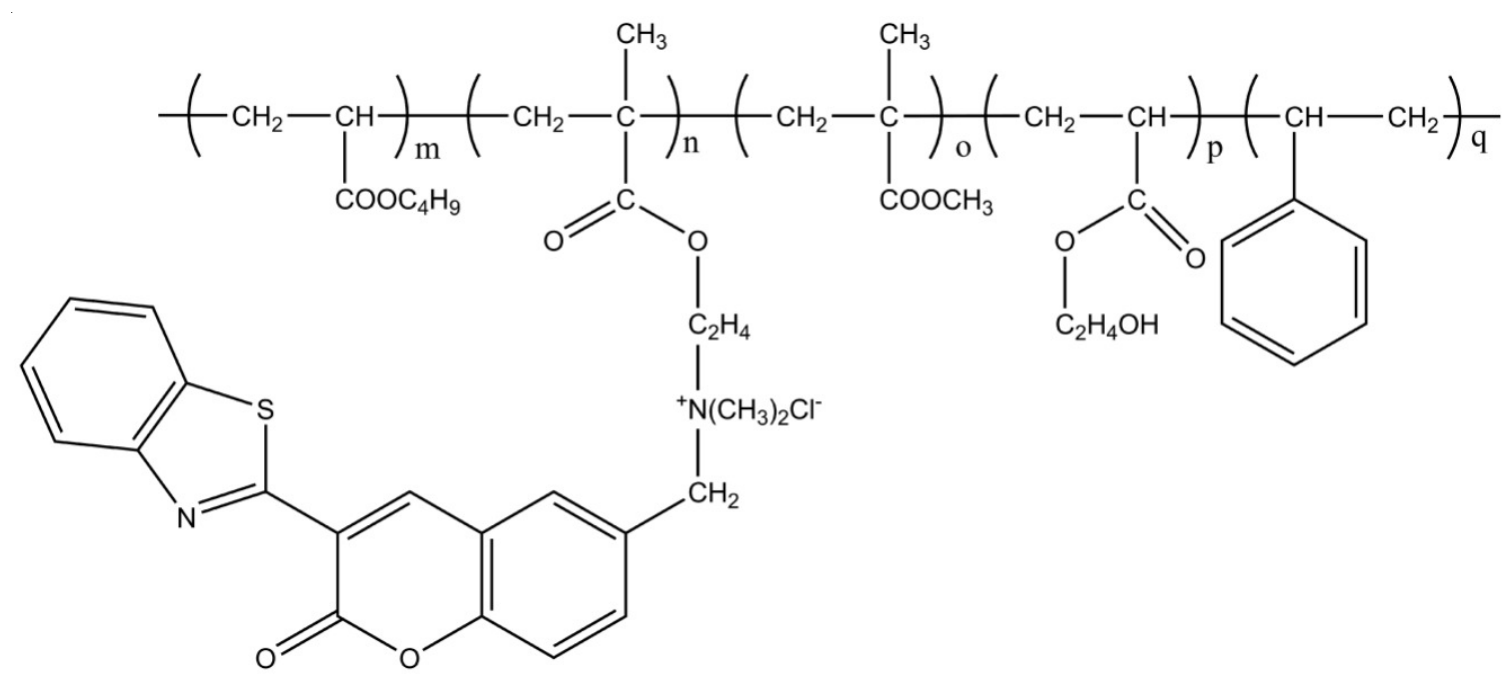

Fig. 1-1. Basic structure of the copolymer C-CPA, by controlling the contents of DM in the polymer, the quantity of fluorophore can be controlled 


\begin{tabular}{ccccccccc}
\hline \multicolumn{7}{c}{ TABLE-1 } \\
\hline \multicolumn{8}{c}{ COMPONENTS USED FOR SYNTHESIZING CPA } \\
\hline $\mathrm{CPA}_{1}$ & IPA $(\mathrm{mL})$ & MMA $(\mathrm{mL})$ & BA $(\mathrm{mL})$ & ST $(\mathrm{mL})$ & HEA $(\mathrm{mL})$ & DM $(\mathrm{mL})$ & AIBN $(\mathrm{g})$ & DM $(\%)$ \\
$\mathrm{CPA}_{2}$ & 70.0 & 25.0 & 38.0 & 13.0 & 18.0 & 10.0 & 1.8 & 9.69 \\
$\mathrm{CPA}_{3}$ & 70.0 & 25.0 & 48.0 & 13.0 & 18.0 & 20.0 & 1.8 & 16.29 \\
\hline
\end{tabular}

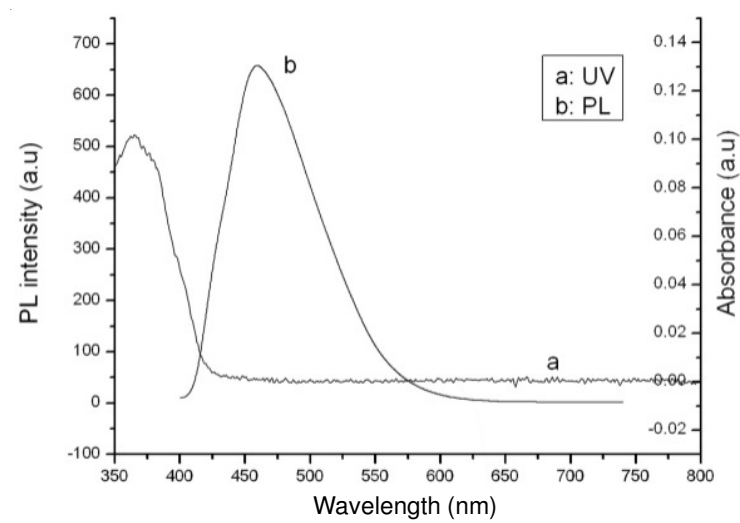

Fig. 1-2. UV spectra and photoluminescence spectra of C-CPA, it shows the polymer has a UV-VIS absorption peak at ca. $365 \mathrm{~nm}$ and a fluorescence emission peak at $c a .458 \mathrm{~nm}$

literature $^{22}$. The average size of CdTe QDs was measured by laser particle size distribution analyzer. The average size was about $5.53 \mathrm{~nm}$. Fig. 2 showed that there were a shoulder peak of UV-VIS absorption spectra at $c a .512 \mathrm{~nm}$ and a fluorescence emission peak at ca. $540 \mathrm{~nm}$.

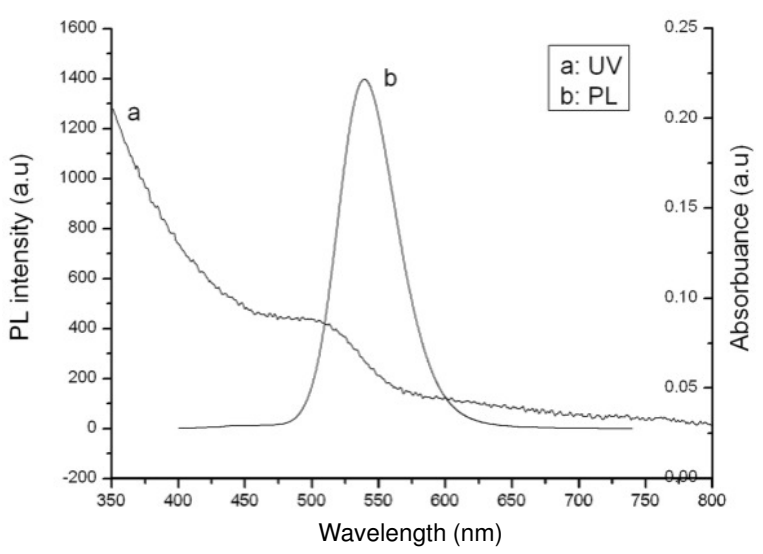

Fig. 2. UV spectra and photoluminescence spectra of CdTe QDs, there as a shoulder peak of UV-VIS absorption spectra at $c a .512 \mathrm{~nm}$ and a fluorescence emission peak at ca. $540 \mathrm{~nm}$

Preparation of C-CPA and CdTe QDs blending system: The series of C-CPA were solved in the solvent of THF and CdTe QDs were solved in water. In order to avoid fluorescent quenching of C-CPA and CdTe QDs caused by their concentration, solutions with a lower concentration were needed to carry out the following researches. Also, for making the fluorescence data be analyzed easily, concentrations of C-CPA and CdTe QDs solutions were controlled to make their fluorescence intensity be of the same order of magnitude. Then, C-CPA $\mathrm{C}-\mathrm{CPA}_{2}$ and $\mathrm{C}-\mathrm{CPA}_{3}$ with a concentration of $0.1 \mathrm{mg} / \mathrm{mL}$ were prepared, CdTe QDs with a concentration of $2 \times 10^{-6} \mathrm{~mol} / \mathrm{mL}$ was prepared as well. These concentrations of the products were the basic concentrations to all the following experiments.
In the following studies, solution of $\mathrm{C}-\mathrm{CPA}_{1}, \mathrm{C}-\mathrm{CPA}_{2}$ and $\mathrm{C}-\mathrm{CPA}_{3}$ were blended with $\mathrm{CdTe}$ solution separately following a certain proportion, then a series of C-CPA and CdTe QDs blending system were prepared.

In the blending system, as C-CPA contented positive charges and CdTe QDs contented negative charges, they attracted each other with electrostatic force. Under the effect of the dipolar interaction, a D-A pair was formed to achieve the energy transfer between these two compounds.

\section{RESULTS AND DISCUSSION}

Variations of photoluminescence properties of both CCPA and CdTe QDs before and after blending: Separately blended CdTe solution with solution of C-CPA ${ }_{1} \mathrm{C}-\mathrm{CPA}_{2}$ and $\mathrm{C}-\mathrm{CPA}_{3}$ with a series of volume ratio in certain total volume of the system, the fluorescence intensity of the blending system was measured with fluorospectrophotometer. The volume fractions of each part of the blending system were showed in Table-2.

\begin{tabular}{|c|c|c|c|c|c|c|c|c|c|}
\hline \multicolumn{10}{|c|}{$\begin{array}{c}\text { TABLE-2 } \\
\text { VOLUME FRACTIONS OF THE ELEMENTS } \\
\text { IN THE BLENDING SYSTEMS }\end{array}$} \\
\hline & 1 & 2 & 3 & 4 & 5 & 6 & 7 & 8 & 9 \\
\hline C-CPA & $5 / 6$ & $4 / 6$ & $2 / 6$ & $1 / 6$ & 0 & $1 / 6$ & $1 / 6$ & $1 / 6$ & $1 / 6$ \\
\hline CdTe QDs & $1 / 6$ & $1 / 6$ & $1 / 6$ & $1 / 6$ & $1 / 6$ & 0 & $2 / 6$ & $4 / 6$ & $5 / 6$ \\
\hline Water & 0 & 0 & 0 & 0 & 0 & $5 / 6$ & $3 / 6$ & $1 / 6$ & 0 \\
\hline THF & 0 & $1 / 6$ & $3 / 6$ & $4 / 6$ & $5 / 6$ & 0 & 0 & 0 & 0 \\
\hline
\end{tabular}

Table-2 showed the contents of each blending system in this part of study. As showed in Table-2, in the first 5 blending systems, the composition of the solvents kept the same and the quantity of CdTe QDs invariable as well, the only variation of the systems was the quantity of C-CPA. Also, the only variation of the last 4 systems was the quantity of CdTe QDs.

In order to make both CdTe QDs and C-CPA have good fluorescence intensity, the excitation wavelength of the fluorospectrophotometer was set at $380 \mathrm{~nm}$. The fluorescence intensity of the blending system with different ratio of CdTe QDs and C-CPA were measured (Fig. 3).

As showed in these figures, all the fluorescence emission peaks of C-CPA appeared at $c a .460 \mathrm{~nm}$ after CdTe aqueous solution was added into C-CPA solution with different volume ratio. Compared with the fluorescence emission peak of simple C-CPA system, the positions of fluorescence emission peak of C-CPA all had a red shift of $c a$. 10-15 nm after CdTe aqueous solution were added. To CdTe QDs, the fluorescence emission peaks of CdTe QDs were appeared at ca. $560 \mathrm{~nm}$ after blended with C-CPA. Compared with the fluorescence emission peak of simple CdTe solution, the positions of fluorescence emission peak of CdTe QDs all had a red shift of $c a .10 \mathrm{~nm}$ after C-CPA when THF solution were added. A further discussion will be taken out later in this paper to explain what factor caused the phenomenon of the red shift. 

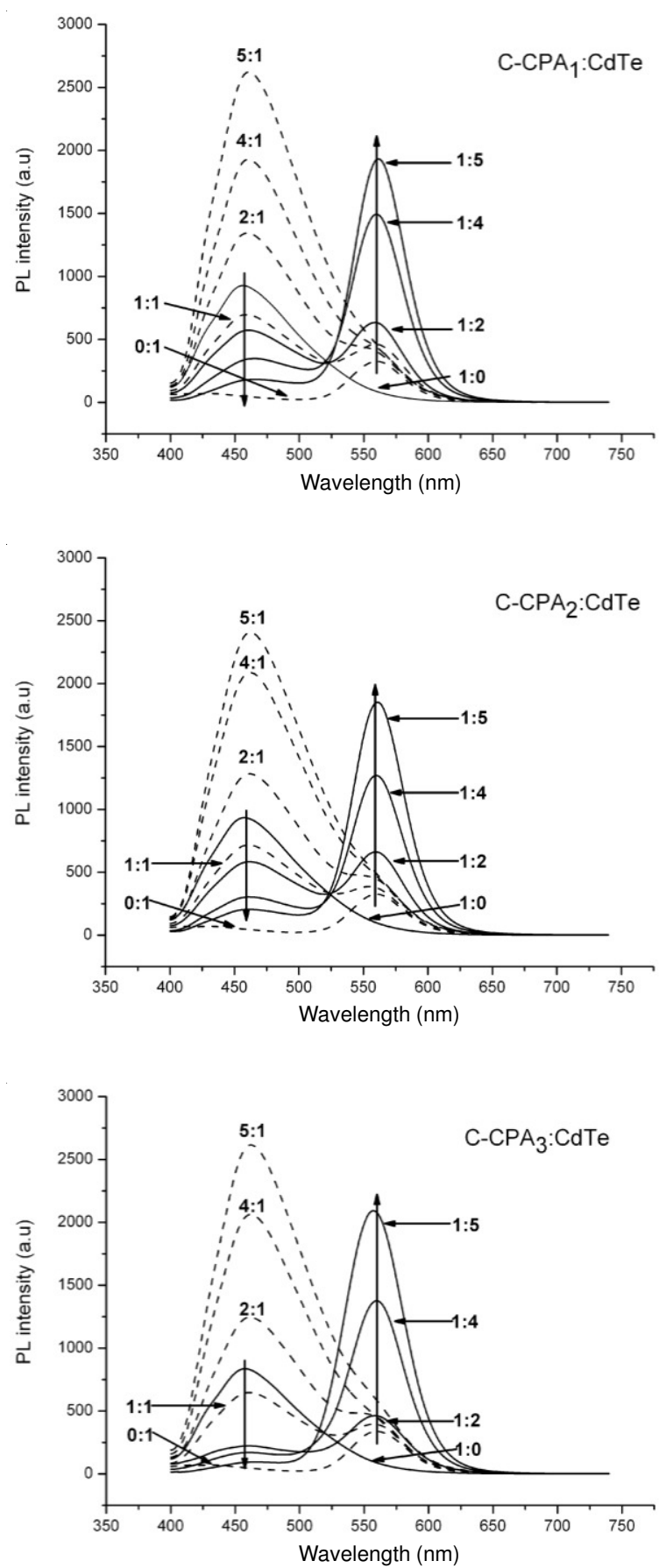

Fig. 3. Fluorescence intensity of the blending system with different ratio of CdTe QDs and C-CPA, the excitation wavelength was set at 380 $\mathrm{nm}$

By analyzing the performance of the photoluminescence properties of C-CPA, it was obvious that the fluorescence intensity of C-CPA varied following not only the variation of its concentration but also variation of the quantity of CdTe QDs added. The volume of the solution haven't been varied in the process and the volume ratio of C-CPA THF solution to CdTe aqueous solution varied from 1:1-1:5, the fluorescence intensity of C-CPA all declined regularly. On the other hand, the fluorescence intensity of CdTe QDs increased with varies degrees after C-CPA was added in.

Variation of photoluminescence properties of C-CPA: In order to show the influences caused by CdTe QDs to the fluorescence intensity of C-CPA clearly, experiments were taken with the volume ratio of C-CPA and CdTe QDs at 1:0, 1:1, 1:2, 1:4 and 1:5 separately and the integrated area ratio of fluorescence emission peak of C-CPA were measured. Supposing that when the volume ratio of C-CPA to CdTe QDs at 1:0, the area of fluorescence emission peak is 1 (Table-3).

\begin{tabular}{cccccc}
\multicolumn{5}{c}{ TABLE-3 } \\
\multicolumn{5}{c}{$\begin{array}{l}\text { INTEGRATED AREA RATIO TO THE } \\
\text { FLUORESCENCE EMISSION PEAK OF C-CPA }\end{array}$} \\
\hline $\mathrm{C}-C P A_{1}$ & $1: 0$ & $1: 1$ & $1: 2$ & $1: 4$ & $1: 5$ \\
$\mathrm{C}-C P A_{2}$ & 1 & 0.76 & 0.66 & 0.44 & 0.24 \\
$\mathrm{C}^{-C P A}$ & 1 & 0.82 & 0.69 & 0.36 & 0.22 \\
& 1 & 0.67 & 0.57 & 0.27 & 0.18 \\
\hline
\end{tabular}

Fig. 4 was drawn with help of the data of Table-3. By analyzing Fig. 4, it can be found that after CdTe QDs were added into C-CPA solution, the integrated area of the fluorescence emission peak of C-CPA with the same concentration declined linearly based on the quantity of CdTe QDs adding into the blending system.

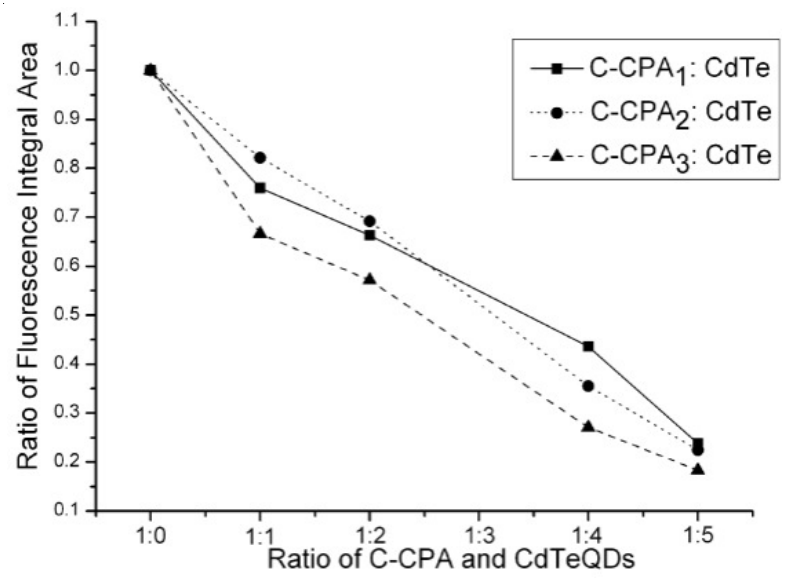

Fig. 4. Relationship between the volume ratio of C-CPA and CdTe QDs and the fluorescence emission peak area of C-CPA

The reason is that C-CPA and CdTe QDs formed a D-A pair which based on the dipolar interaction in the blending system $^{23}$. When C-CPA works as the energy donor and CdTe QDs works as the energy accepter, the degree of energy transfer between these two compounds will increase along with the quantity increasing and this made the fluorescence intensity of C-CPA declined linearly with the quantum increase of CdTe QDs.

Variation of photoluminescence properties of CdTe QDs: In order to further study on the influences caused by adding C-CPA to the photoluminescence properties of $\mathrm{CdTe}$ QDs, a series of blank experiments exclude C-CPA were taken out, the fluorescence intensity of CdTe QDs was measured and the results were compared with former researches. The volume fractions of each part of the blending system are given in Table-4 (Fig. 5).

As showed in the figures above, the fluorescence intensity of CdTe QDs all increased with varying degrees after C-CPA were adding into the CdTe solution with different quantity of CdTe QDs. In order to measure the influences caused by CCPA to the photoluminescence properties of CdTe QDs exactly, 


\begin{tabular}{lcccccccc}
\hline \multicolumn{10}{c}{ TABLE-4 } \\
\multicolumn{10}{c}{ VOLUME FRACTIONS OF THE ELEMENTS IN } \\
THE BLENDING SYSTEMS \\
\hline \multicolumn{1}{c}{1} & 2 & 3 & 4 & 5 & 6 & 7 & 8 \\
\hline C-CPA & 0 & 0 & 0 & 0 & $1 / 6$ & $1 / 6$ & $1 / 6$ & $1 / 6$ \\
CdTe QDs & $5 / 6$ & $4 / 6$ & $2 / 6$ & $1 / 6$ & $5 / 6$ & $4 / 6$ & $2 / 6$ & $1 / 6$ \\
Water & 0 & $1 / 6$ & $3 / 6$ & $4 / 6$ & 0 & $1 / 6$ & $3 / 6$ & $4 / 6$ \\
THF & $1 / 6$ & $1 / 6$ & $1 / 6$ & $1 / 6$ & 0 & 0 & 0 & 0 \\
\hline
\end{tabular}
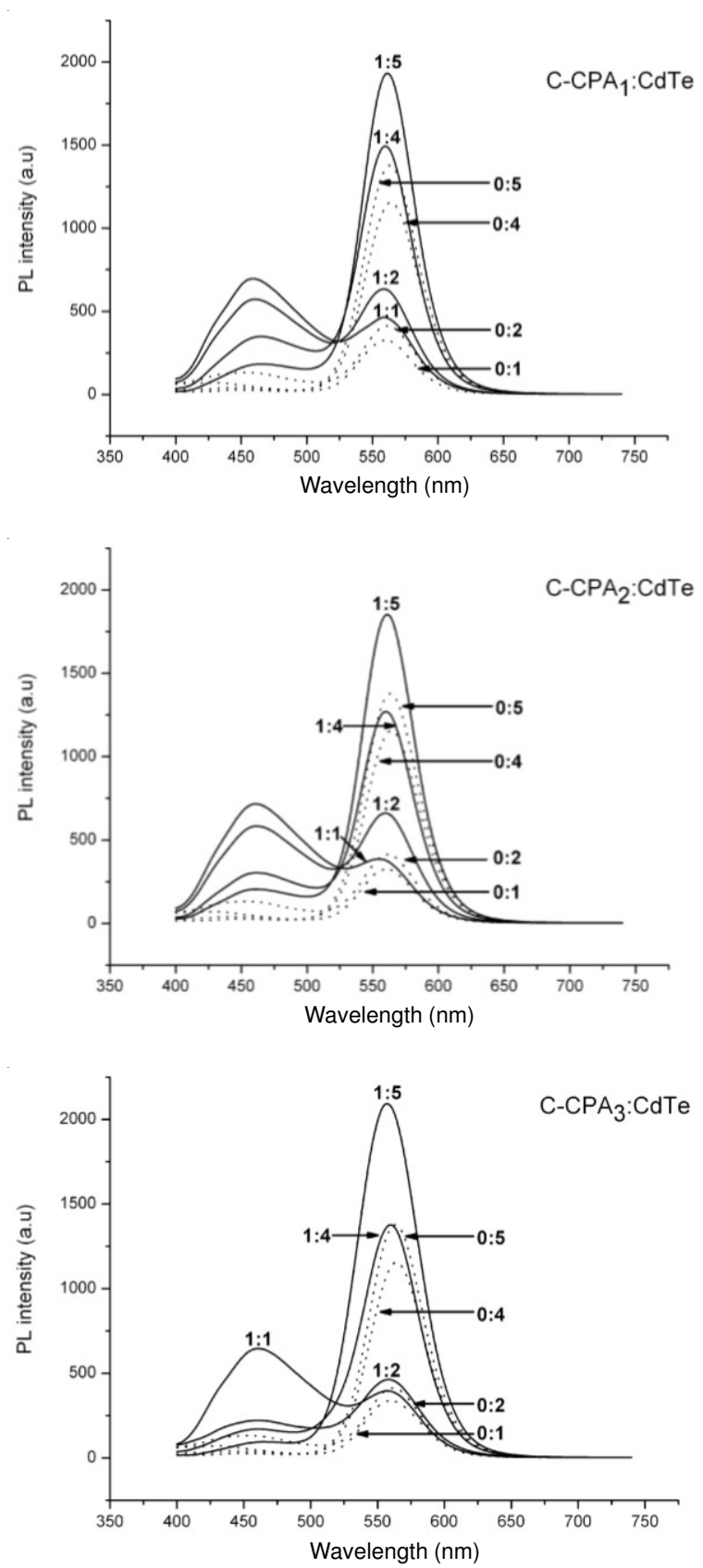

Fig. 5. Fluorescence intensity of CdTe QDs with and without C-CPA added in

UV-VIS spectrophotometer was used to measure the UV absorption intensity of CdTe QDs with different concentrations at $380 \mathrm{~nm}$ and the fluorescence emission peak areas of CdTe QDs were calculated as well. Then the quantum yields of CdTe QDs were computed by the formula (1):

$$
\Phi_{\mathrm{x}}=\frac{\mathrm{n}_{\mathrm{x}}^{2}}{\mathrm{n}_{\mathrm{i}}^{2}} \cdot \frac{\mathrm{A}_{\mathrm{i}} \mathrm{D}_{\mathrm{x}}}{\mathrm{A}_{\mathrm{x}} \mathrm{D}_{\mathrm{i}}} \Phi_{\mathrm{i}}
$$

where $A_{x}$ and $A_{i}$ are the absorbencies at the excitation wavelength of the sample and the reference, respectively. $D_{x}$ and $D_{i}$ are the integrated fluorescence intensities of the sample and the reference, respectively. The $n_{x}$ and $n_{i}$ are the refractive indexes of the sample and the reference, respectively and $\Phi_{\mathrm{x}}$ and $\Phi_{\mathrm{i}}$ are the quantum yields (QYs) of the sample and the reference, respectively.

The experiment took rhodamine $6 \mathrm{G}$ as photoluminescence reference to calculate the quantum yields of CdTe QDs according to the formula (1). The result showed that the quantum yields of the simple CdTe QDs was ca. $13.5 \%$. Then the quantum yields of the simple CdTe QDs was taken as reference and the quantum yields of CdTe QDs in the blending systems before and after different quantity of C-CPA added in were calculated (Table-5, Fig. 6).

\begin{tabular}{|c|c|c|c|c|c|c|c|c|}
\hline \multicolumn{9}{|c|}{$\begin{array}{c}\text { TABLE-5 } \\
\text { TAKE THE QUANTUM YIELD OF THE SIMPLE CdTe QDs } \\
\text { AS } 1 \text { AND CALCULATE THE QUANTUM YIELDS OF } \\
\text { CdTe QDs WITH OR WITHOUT C-CPA BLENDING IN }\end{array}$} \\
\hline & $0: 1$ & $0: 2$ & $0: 4$ & $0: 5$ & $1: 1$ & $1: 2$ & $1: 4$ & $1: 5$ \\
\hline C-CРA & 1 & 1.00 & 1.22 & 1.16 & 1.62 & 1.76 & 1.58 & 1.65 \\
\hline C-CPA ${ }_{2}$ & 1 & 1.00 & 1.23 & 1.19 & 1.92 & 1.94 & 2.08 & 2.25 \\
\hline $\mathrm{C}-\mathrm{CPA}_{3}$ & 1 & 0.97 & 1.22 & 1.15 & 2.21 & 2.23 & 2.67 & 2.97 \\
\hline
\end{tabular}

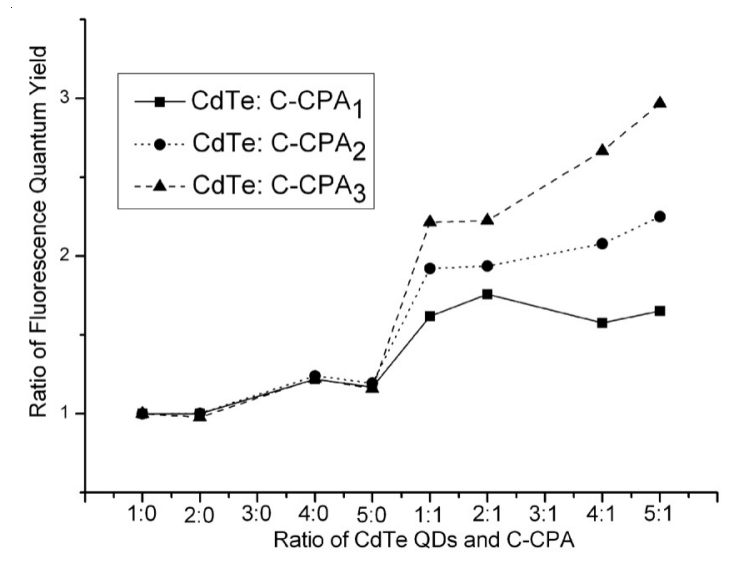

Fig. 6. Relationship between the the volume ratio of C-CPA and CdTe QDs and the quantum yields of CdTe QDs

As showed in Table-5 (Fig. 6), the quantum yields of CdTe QDs varied little before C-CPA adding into the blending system. This phenomenon conform the theory that the concentration will have little affect to the quantum yields of the fluorescent substance as the concentration of the fluorescent substance is low enough ${ }^{24}$. After C-CPA added into the blending system, the quantum yields of CdTe QDs with different concentrations all increased more than $50 \%$. This phenomenon showed that the addition of C-CPA largely enhanced the quantum yields of CdTe QDs.

Then, the influences caused by $\mathrm{C}-\mathrm{CPA}_{1} \mathrm{C}-\mathrm{CPA}_{2}$ and $\mathrm{C}$ $\mathrm{CPA}_{3}$ to the quantum yields of CdTe QDs were tested. After $\mathrm{C}-\mathrm{CPA}_{1}$ added into the blending system, the quantum yields of CdTe QDs all increased about $60 \%$, but as the quantity of CdTe QDs increasing, the quantum yields of CdTe QDs decreased a little. After C-CPA 2 added into the blending system, the quantum yields of CdTe QDs increased $c a .100$ $\%$. Along with the quantity increase of CdTe QDs, the quantum 
yields of CdTe QDs has a minor increase. After C-CPA 3 added, the quantum yields of CdTe QDs all increased more than $100 \%$ and as the quantity of CdTe QDs increasing, the quantum yields also increased obviously. From the experiments, it was inferred that the contents of coumarin and positive charges on the polymer were the main factors which affected the photoluminescence properties of CdTe QDs. The energy transfer between C-CPA and CdTe QDs followed the fluorescence resonance energy transfer theory (FRET) ${ }^{23,25,26}$, the formula (2) shows the factors which affect the rate constant of FRET:

$$
\mathrm{k}_{\mathrm{et}}=\frac{\mathrm{c} \Phi_{\mathrm{e}} \mathrm{K}^{2}}{\mathrm{n}^{4} \tau \mathrm{d}^{6}} \int \frac{\mathrm{f}_{\mathrm{d}}(v) \varepsilon_{\mathrm{A}}(v) \mathrm{d} v}{v^{4}}
$$

where $\Phi_{\mathrm{e}}$ and $\tau$ are the quantum yields and lifetime of excited state to the excitation donor; $\varepsilon$ is extinction coefficient; $\mathrm{K}$ is orientational factor of optical transitions, in the situation of random orientation, the value is $2 / 3$; $d$ is the distant between the donor and accepter. The integral quantity equals to the overlapping area of the fluorescence emission peak of the donor and the fluorescence excitation peak of the accepter. As the donor in this part of research didn't have any change, the value of $\Phi_{\mathrm{e}}$ and $\tau$ will not be changed, the distant of the donor and accepter have a tremendous impact to the value of $k_{\mathrm{et}}$. At the same time, the concentration of the accepter and the overlapping area of the fluorescence emission peak of the donor and the fluorescence excitation peak of the accepter also have some influences to the value of $k_{\text {et }}$. In present research work, the fluorescence excitation peak didn't varied either and from the spectrum showed in Fig. 5, the concentration of CdTe QDs was the direct factor that affected the overlapping area. In brief, $\mathrm{d}$ and $\mathrm{c}$ in formula (2) were the main effect factors in this part of research.

With the formula (2), a series of conclusions might be got. In the situation that $\mathrm{CdTe}$ QDs and C-CPA 1 blended together, the contents of coumarin and positive charges were low and the electrostatic force didn't promoted much as the concentration of CdTe QDs increasing, the dipolar interaction between CdTe QDs and C-CPA 1 was limited, so the quantum yields of CdTe QDs in this situation didn't varied much with the concentration variation of $\mathrm{C}-\mathrm{CPA}_{1}$. In the situation that CdTe QDs and C-CPA 2 blended together, the contents of coumarin and positive charges were increased and the quantum yields of CdTe QDs generally consistent with formula (2), electrostatic interaction between CdTe QDs and C-CPA increased comparing with the former situation, along with the concentration increasing of CdTe QDs, the quantum yields of CdTe QDs increased as well. In the situation that CdTe QDs and $\mathrm{C}-\mathrm{CPA}_{3}$ blended together, the contents of coumarin and positive charges were further increased. It made a stronger electrostatic interaction between these two components of the blending system and made the distant between these two components closer. So the quantum yields of CdTe QDs have a trend of further increase and the trend consistent with the formula (2).

Affects caused by solvent to the photoluminescence properties of CdTe QDs: Comparing the photoluminescence properties of CdTe QDs in aqueous solution with its photoluminescence properties in the blending system, it was remarkable phenomenon that the fluorescence emission peak of CdTe QDs had a red shift after C-CPA THF solution adding into the system, when the quantity of CdTe QDs in the system increasing, the tendency of red shift declined. Considering that the position of fluorescence emission peak of CdTe QDs based on its particle size, laser scattering particle analyzer was used to measure the average particle size. The results were showed in Fig. 7.
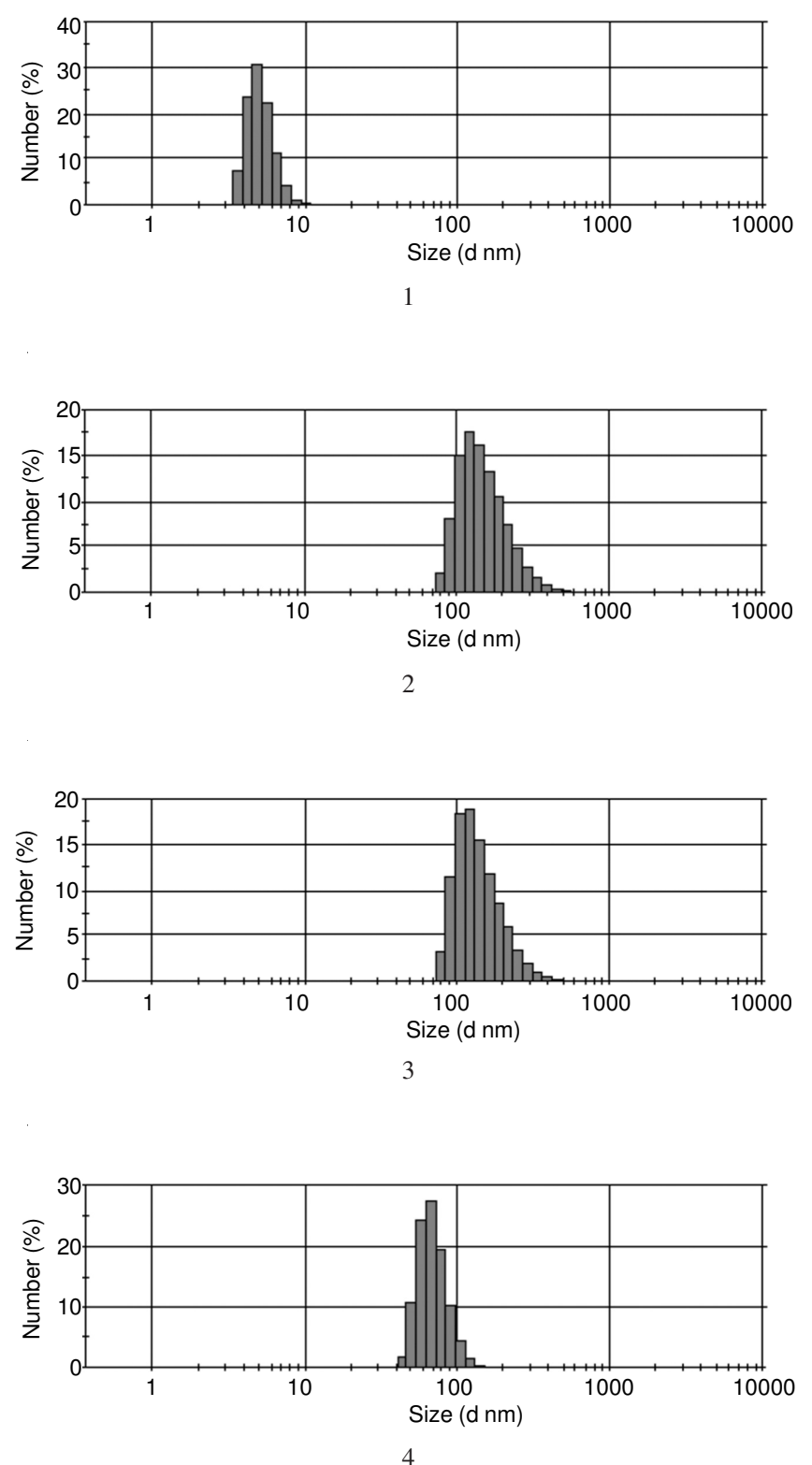

Fig. 7. Average particle size of CdTe QDs measured by laser scattering particle analyzer

Calculating the data got from the figures above. The average particle size of CdTe QDs in aqueous solution was ca. $5.53 \mathrm{~nm}$ (Fig. 7-1); The average particle size of CdTe QDs all over $50 \mathrm{~nm}$ in the blending system with the solvent of water and THF at a certain volume ratio. For analyzing the particle size variation of CdTe QDs, a series of compare experiments with the CdTe QDs in different situations were taken. First, an experiment to test the average particle size of CdTe QDs blended with $\mathrm{C}-\mathrm{CPA}_{2}$ THF solution at the volume ratio of 1:1 compared with the particle size of CdTe QDs in the same solvents environment but without the addition of $\mathrm{C}-\mathrm{CPA}_{2}$ was 
taken. It was found that when $\mathrm{C}-\mathrm{CPA}_{2}$ was added in, the average particle size of CdTe QDs was ca. $160.01 \mathrm{~nm}$ (Fig. 72) and in the situation of no C-CPA $\mathrm{C}_{2}$ was added in, the average particle size was ca. $160.14 \mathrm{~nm}$ (Fig. 7-3). So it can be inferred that the addition of C-CPA was not the main factor affected the particle size of CdTe QDs. Then the average particle size was researched that when the quantity of CdTe QDs were different and it was found when the volume ratio of CdTe aqueous solution and $\mathrm{C}-\mathrm{CPA}_{2}$ THF solution was at 5:1, the average particle size of CdTe QDs was about only $60.40 \mathrm{~nm}$ (Fig. 74). This showed that the quantity of CdTe QDs in the blending system affected the average particle size of CdTe QDs significantly. Considering all the phenomena above, THF can be inferred as the main factor caused the particle size variation of CdTe QDs ${ }^{27}$. When THF added in the system, no matter $\mathrm{C}$-CPA was added or not added in the system, the particle size of CdTe QDs all changed and as the ratio of THF increasing, the affect increased as well. At the same time, if the addition of THF really had such a significant affect to the size of CdTe QDs, the fluorescence emission peak of CdTe QDs will also drifted significantly as the position of fluorescence emission peak of CdTe QDs was determined by its particle size. But comparing the fluorescence spectrogram of CdTe QDs in aqueous solution and in the blending system, the position drift of the fluorescence emission peak was not so obviously. This contradiction showed a probable explanation that the addition of THF didn't changed the particle size of CdTe QDs, but after THF was added into the system, the distribution pattern of CdTe QDs in solution has been changed compared with the situation of the aqueous solution, THF reduced its dispersibility. So in the blending system, the positions of fluorescence emission peak of CdTe QDs all have a red shift of $c a$. $10 \mathrm{~nm}$ and the red shift decreased when the quantity of CdTe QDs increasing.

\section{Conclusion}

Based on the experiments and the researches we have done, we may conclude the following. A series of positive charged C-CPA with different contents of coumarin and a negative charged CdTe QDs protected by TGA were synthesized. Both of these compounds have good photoluminescence properties. When blending them in a solution system, the stability of the mixtures didn't decline. In the blending system, the fluorescence intensity of C-CPA varied following the quantity of CdTe QDs added. With the quantity of CdTe QDs increase, the fluorescence intensity of C-CPA all declined regularly. On the other hand, the fluorescence intensity of CdTe QDs increased with varies degrees after C-CPA was added in. The fluorescence intensity of C-CPA declined linearly based on the quantity of CdTe QDs adding into the blending system; the fluorescence intensity of CdTe QDs increased based on the quantity of C-CPA and the contents of coumarin connected on the copolymer. These phenomena showed that there may be an energy transfer in the blending system between these two mixtures. As there were different charges in the two compounds, electrostatic force were inferred as the main driving force of energy transfer. The data of fluorescence intensity of CdTe QDs blended with different kind of C-CPA can be explained by the theory FRET and a conclusion that C-CPA and CdTe QDs formed an energy donor-acceptor pair which based on the dipolar interaction in the blending system was got. By using laser scattering particle analyzer to analyze the average particle size of CdTe QDs, we found THF has reduced the dispersibility CdTe QDs and this made the positions of fluorescence emission peak of CdTe QDs all have a red shift.

\section{ACKNOWLEDGEMENTS}

Financial support from the Science and Technology Department of Sichuan (No. 2011FZ0107) is greatly appreciated.

\section{REFERENCES}

1. J.K. Ryu and C.B. Park, Biotechnol. Bioeng. (2009).

2. P. Fortina, L.J. Kricka, S. Surrey and P. Grodzinski, Trends Biotechnol., 23, 4 (2005).

3. X. Hou, Q. Li, L. Jia, Y. Li, Y. Zhu and A. Cao, Macromol. Biosci., 9, 551 (2009).

4. Y.Q. Mao, N. Li, Y. Han, B.A. Ning, Z.X. Gao and Z.J. Zhou, Asian J. Chem., 24, 3715 (2012).

5. K. Shrivas, S.K. Kailasa and H.-F. Wu, Proteomics, 9, 2656 (2009).

6. M. Bosi and C. Pelosi, Prog. Photovolt, Res. Appl., 15, 51 (2007).

7. N. Daldosso and L. Pavesi, Laser Photon Rev., 3, 508 (2009).

8. W.U. Huynh, J.J. Dittmer and A.P. Alivisatos, Science, 295, 2425 (2002).

9. K.L. Chopra, P.D. Paulson and V. Dutta, Prog. Photovolt: Res. Appl., 12, 69 (2004).

10. P.B. Yim, M.L. Clarke, M. McKinstry, S.H. De Paoli Lacerda, L.F. Pease 3rd, M.A. Dobrovolskaia, H. Kang, T.D. Read, S. Sozhamannan and J. Hwang, Biotechnol. Bioeng., 104, 1059 (2009).

11. X.Y. Wang, Q. Ma, B. Li, Y.B. Li and X.G. Su, Luminescence, 22, 1 (2007).

12. T. Nakashima, Y. Nonoguchi and T. Kawai, Polym. Adv. Technol., 19, 1401 (2008).

13. H. Zhang, Z. Cui, Y. Wang, K. Zhang, X. Ji, C. Lü, B. Yang and M. Gao, Adv. Mater., 15, 777 (2003).

14. X.D. Cao, C.M. Li, H.F. Bao, Q.L. Bao and H. Dong, Chem. Mater., 19, 3773 (2007).

15. J. Pan, Y. Wang and S.S. Feng, Biotechnol. Bioeng., 101, 622 (2008).

16. C. Bertoni, D. Gallardo and S. Dunn, Appl. Phys. Lett., 90, 034107 (2007).

17. Y. Xuan, G. Qian, Z.Y. Wang and D.G. Ma, Thin Solid Films, 516, 7891 (2008).

18. R. He, X.-F. Qian, J. Yin, L.-J. Bian, H.-A. Xi and Z.-K. Zhu, Mater. Lett., 57, 1351 (2003).

19. H. Sun, J. Zhang, Y. Tian, Y. Ning, H. Zhang, J. Ju, D. Li, S. Xiang and B. Yang, J. Nanomater., Article ID 38589 (2007).

20. Y.F. Liu and J.S. Yu, J. Colloid Interf. Sci., 333, 690 (2009).

21. C.Z. Zhang, R.Y. Liang, C.X. Jiang, D.B. Chen and A.Y. Zhong, J. Appl. Polym. Sci., 108, 2667 (2008).

22. H. Zhang, L. Wang, H. Xiong, L. Hu, B. Yang and W. Li, Adv. Mater., 15, 20 (2003).

23. K. Okawa, T. Miyata and T. Uragami, J. Polym. Sci.: B: Polym. Phys., 44, 3245 (2006).

24. M.1 Frigoli, K. Ouadahi and C. Larpent, Chem. Eur. J., 15, 8319 (2009).

25. L. Bene, G. Szentesi, L. Mátyus, R. Gáspár and S. Damjanovich, J. Mol. Recogn., 18, 236 (2005).

26. H.-Q. Wang, Y.-Q. Li, J.-H. Wang, Q. Xu, X.-Q. Li and Y.-D. Zhao, Anal. Chim. Acta, 610, 68 (2008).

27. S. Si, E. Dinda and T.K. Mandal, Chem. Eur. J., 13, 9850 (2007). 